\title{
A STUDY TO DETERMINE CO-RELATION BETWEEN DISEASE BURDEN, NUMBER OF CLINICAL TRIALS DONE AND SUCCESS RATES FOR GERMANY AND INDIA.
}

\author{
Dnyanesh Limaye ${ }^{1}$, Gerhard Fortwengel ${ }^{2}$, Vaidehi Limaye ${ }^{3}$
}

\author{
HOW TO CITE THIS ARTICLE: \\ Dnyanesh Limaye, Gerhard Fortwengel, Vaidehi Limaye. "A Study to Determine Co-Relation between \\ Disease Burden, Number of Clinical Trials Done and Success Rates for Germany and India". Journal of \\ Evidence based Medicine and Healthcare; Volume 1, Issue 17, December 29, 2014; Page: 2186-2196.
}

ABSTRACT: The drugs we use to treat any condition - from an innocuous cough to a lifethreatening cancer - are the outcome of painstaking human clinical trials. These trials are the only way to credibly determine the safety and efficacy of drugs. In recent years there has been a clear shift in clinical trial sites from core developed countries like USA, European countries to developing countries like India, China, South American countries. This shift is related to challenges and opportunities like costs of trials, recruitment issues, and regulatory challenges in developed vs. developing countries. Developing countries and developed countries have their unique disease burden patterns based on various parameters like but not limited to age, health care facilities, health insurance, sanitary conditions, environmental issues, education, nutrition and GDP. Previous studies have reported that many of the important global diseases are not much explored in clinical trials and many published clinical trials have very less international health relevance. This study was aimed at finding the correlation between disease burdens, number of clinical trials done and trial success rates. We compared 2005-2010 Global Burden of Disease data for Germany, India and number of clinical trials from clinicaltrials.gov database done in the same period. Our findings indicated that there was a good correlation between the disease burden and clinical trials for Germany in 2005 and 2010. For India in 2005 there was a moderate positive correlation, 2010 data showed the improvement in India in terms of match between disease burden and clinical Trials. But careful observation of the data shows still a need for more trials on Communicable, maternal, neonatal and nutritional disorders.

KEYWORDS: Disease Burden, Clinical Trials, Developing Countries, India, Germany, DALY.

INTRODUCTION: More than two-thirds of the world's population live in low-income countries, where health priorities are very different from those in more affluent parts of the world. Relative to people living in wealthy nations, the most impoverished $20 \%$ of the world's population is 9 times more likely to die of infectious diseases and 10 times more likely to die in childhood. ${ }^{1}$

Developed country like Germany has unique pattern of diseases, where due to increasing life expectancy chronic diseases account for $92 \%$ of all deaths. The Federal Government in Germany has taken steps to establish German Centers for Health Research to pool research into a number of particularly important common diseases in Germany. The developing country like India has its own problems because of wide gap between the rich and poor population. It has diseases of both developing and developed countries. ${ }^{2}$ 
On an international level, the Global Forum for Health Research has estimated that less than $10 \%$ of health research spending is directed toward diseases or conditions that account for $90 \%$ of the global burden of disease, a phenomenon referred to as the "10/90 gap." These numbers suggest that many common conditions or diseases are not being adequately studied.

Numerous studies have found that the drug development process is highly expensive and that these costs have trended significantly upward for decades. The clinical approval success rate in the United States was 16\% to $19 \%$ during both the 1993-1998 and the 1999-2004 sub periods. However success rates varied significantly by therapeutic class. Clinical approval success rates varied from $8 \%$ for CNS drugs to $24 \%$ for systemic anti-infectives. ${ }^{1}$ Newer clinical trial designs like adaptive clinical trials have been suggested to improve the clinical trial success rates. Since the early 1990s, growth in the number of people participating in and required for pharmaceutical clinical trials has been massive. The number of clinical trial investigators conducting multinational drug research in low-income settings increased 16-fold in the past decade (Office of Inspector General, Department of Health and Human Services 2001).

Many of these new trials are being performed in geographical areas of political and economic instability and unprecedented health care crises and where subjects are readily accessible. Drug companies' apparent ease of accessibility to such areas raises questions about the unequal social contexts in which research is being performed and about how conditions of inequality are at present facilitating a global proliferation of pharmaceutical drug trials. ${ }^{3}$

If we wish to understand what exactly drives the demand for larger pools of human subjects? First, it is the sheer number of trials being run. As of 2000, there were about 7,500 new clinical projects being designed for research and development worldwide. By 2001, that number had purportedly grown to 10,000 . Second, to satisfy U.S. regulatory demands, increasingly large numbers of patients must be included in clinical trials to prove products' long-term safety, especially for drugs intended to be widely prescribed. Third, some therapeutic categories-such as hypertension-are being overwhelmed with new drugs. Competition to get these drugs approved and to bring them to market intensifies the search for subjects. Fourth, there is a "drug pipeline explosion"-patent applications are flooding the U.S. Patent Office for new compounds that have yet to be clinically tested. ${ }^{3}$

Shifts in the very science of drug development also influence the decision to increase subject recruitment. As a vast amount of potential molecular therapeutics is generated, making right decisions regarding which molecules to test becomes more difficult. Wall Street investors learned that when the technology showed signs of failure in a late-phase clinical trial for patients with skin cancer, researchers recruited more research subjects in an attempt to find a statistically significant positive result. Finally, the available pool of human subjects in the United States is shrinking. The relatively affluent U.S. population is using too many drugs. "Treatment saturation" is making Americans increasingly unusable from a drug-testing standpoint, as pharmaceuticalized bodies produce too many drug - drug interactions, providing less and less capacity to show drug effectiveness and making test results less statistically valid. ${ }^{3}$

Developing country like India which was on the radar of all the pharma companies as well as CRO's has recently taken steps, regulatory amendments to protect the rights of people, and to ensure ethical conduct of clinical trials. 
After taking a review of all the above information it is very important to know the correlation between disease burdens, number of clinical trials done and success rates of these trials. This study will help to gain following understanding of a much sought co-relation between disease burden and number of clinical trials done. Looking at the increasing number of clinical trials done in India It will help to answer the important question do we really need these trials, is it ethical, does it fall in line with typical health situation in India - a country with disease of both developing and developed countries? It will also help to understand which disease areas need more clinical research in a developed country like Germany with increasing aging population and subsequent increase in age related diseases. Finally this study will offer some suggestions to a unique problem of decreasing success of clinical trials.

\section{OBJECTIVES:}

- To evaluate whether the number of clinical trials conducted on various medical conditions are related to the burden of disease and health needs of the local populations in Germany and India in the time period of 2005-2013.

- To determine the success rates of clinical trials conducted on various medical conditions in Germany and India from 2005 - 2010.

\section{MAIN OUTCOME MEASURES:}

Countries: Germany and India; Time Period: 2005-2010.

- Number of trials and randomized subjects for each category of disease in the global burden of disease taxonomy.

- Disease burden analysis.

- Phase-wise trial success rates for each category of disease.

MATERIALS AND METHODS: We compared 2005-2010 Global Burden of Disease data for Germany, India and number of clinical trials from clinicaltrials.gov database done in the same period. ${ }^{4,5}$

To assess the burden of disease, both mortality and morbidity must be taken into account. We used disability-adjusted life year (DALY) as a measure of burden of disease. The DALY is a time-based measure that combines years of life lost due to premature mortality and years of life lost due to time lived in health states of less than ideal health.

Mortality is based only on the number of deaths, whereas as DALYs for a disease or health condition are calculated as the sum of the Years of Life Lost (YLL) due to premature mortality in the population and the Years Lost due to Disability (YLD) for people living with the health condition or its consequences. (WHO Reference)

We used a careful 2-step process for data search of this study. First we conducted an extensive search from GBD database (Reference) for the top 25 diseases for Germany and India in the period from 2005 to 2010. As a second step for those top 25 diseases for Germany and India we did search to determine the number of trials specifically for Germany and India from clinicaltrials.gov database in the period of 2005 to 2010. 
DALY values were then expressed as \% DALY based on total DALY (All cause), on the similar basis clinical trial for each of the 25 diseases were expressed as \% clinical trials based on total clinical trial for all 25 diseases for ease of analysis.

Correlation coefficient R was then calculated for \% DALY and \% Clinical Trials for 2005 and 2010. $P$ value was also determined to understand the significance of the result.

RESULTS: Table 1 shows the values of \% of DALYS and \% Clinical trials done in Germany in 2005 for top 25 diseases in Germany. ${ }^{4,5}$ This table clearly shows that Non communicable diseases $(87.9 \%)$, Cardiovascular and circulatory diseases (19.9\%) and Neoplasms (17.8\%) are the top 3 diseases in this period. Table 1 shows that in this period there is a good match between \% DALYs and \% Clinical trials.

\begin{tabular}{|c|c|c|c|}
\hline Location & Cause & \% DALYS & $\%$ clinical trials \\
\hline Germany & Total (All Causes) & 100.0 & 100.0 \\
\hline Germany & Non-communicable diseases & 87.9 & 94.7 \\
\hline Germany & Cardiovascular and circulatory diseases & 19.9 & 28.6 \\
\hline Germany & Neoplasms & 17.8 & 32.2 \\
\hline Germany & Musculoskeletal disorders & 15.6 & 4.4 \\
\hline Germany & Low back and neck pain & 11.4 & 0.4 \\
\hline Germany & Mental and behavioral disorders & 11.4 & 5.8 \\
\hline Germany & Ischemic heart disease & 11.0 & 14.5 \\
\hline Germany & Low back pain & 8.9 & 0.1 \\
\hline Germany & Injuries & 8.6 & 0.0 \\
\hline Germany & Diabetes, urogenital, blood, and endocrine diseases & 5.9 & 8.4 \\
\hline Germany & Other non-communicable diseases & 4.9 & 5.2 \\
\hline Germany & Neurological disorders & 4.5 & 4.9 \\
\hline Germany & Unipolar depressive disorders & 4.4 & 1.0 \\
\hline Germany & Chronic respiratory diseases & 4.3 & 3.4 \\
\hline Germany & Unintentional injuries other than transport injuries & 4.3 & 0.3 \\
\hline Germany & Cerebrovascular disease & 4.1 & 2.7 \\
\hline Germany & Major depressive disorder & 3.8 & 1.0 \\
\hline Germany & Trachea, bronchus, and lung cancers & 3.6 & 4.1 \\
\hline Germany & Communicable, maternal, neonatal, and nutritional disorders & 3.5 & 10.0 \\
\hline Germany & Falls & 3.2 & 0.3 \\
\hline Germany & Chronic obstructive pulmonary disease & 2.8 & 1.3 \\
\hline Germany & $\begin{array}{r}\text { Diabetes mellitus } \\
\end{array}$ & 2.7 & 5.7 \\
\hline Germany & Neck pain & 2.5 & 0.1 \\
\hline Germany & Ischemic stroke & 2.5 & 2.7 \\
\hline Germany & Other musculoskeletal disorders & 2.5 & 0.0 \\
\hline
\end{tabular}

Table 1: Germany 2005, \% DALY and \% Clinical trials 
Graph 1 shows the correlation between \% DALYs and \% Clinical trials. We calculated Pearson Correlation Coefficient and the value of $R$ is 0.9559 . This shows a strong positive correlation, which means that high \% DALYs variable scores go with high \% Clinical trials scores (and vice versa). $P$ Value from Pearson $(R)$ is $<0.00001$. The result is significant at $p<0.05$.

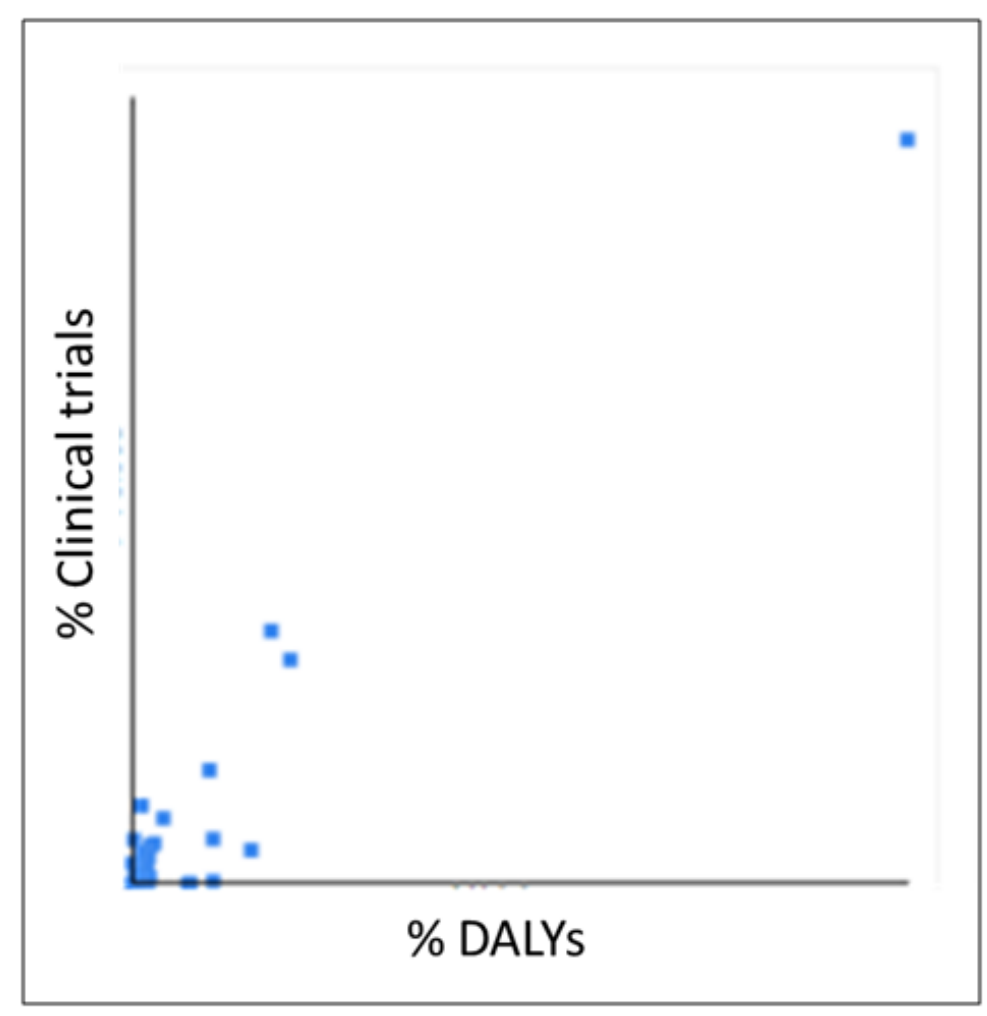

\section{Graph 1: Correlation between \% DALYs and $\%$ Clinical Trials for Germany 2005}

Table 2 shows the values of \% of DALYS and \% Clinical trials done in India in 2005 for top 25 diseases in India. ${ }^{4,5}$ This table clearly shows that Communicable, maternal, neonatal, and nutritional disorders (48.4\%), Non communicable diseases (40.6\%) and Diarrhea, lower respiratory infections, meningitis, and other common infectious diseases (18.2\%) are the top 3 diseases in this period. 


\begin{tabular}{|c|c|c|c|}
\hline Location & Cause & $\%$ DALYS & $\%$ clinical trials \\
\hline India & Total (All Causes) & 100 & 100 \\
\hline India & Communicable, maternal, neonatal, and nutritional disorders & 48.4 & 28.6 \\
\hline India & Non-communicable diseases & 40.6 & 91.7 \\
\hline India & $\begin{array}{r}\text { Diarrhea, lower respiratory in fections, men ingitis, and other } \\
\text { common infectious diseases }\end{array}$ & 18.2 & 6.3 \\
\hline India & Neonatal disorders & 14.0 & 3.6 \\
\hline India & Injuries & 11.0 & 0.0 \\
\hline India & Cardiovascular and circulatory diseases & 8.6 & 26.6 \\
\hline India & Diarrheal diseases & 6.1 & 3.1 \\
\hline India & Chronic respiratory diseases & 5.9 & 4.2 \\
\hline India & Lower respir atory infections & 5.9 & 1.0 \\
\hline India & Preterm birth complications & 5.7 & 0.0 \\
\hline India & Unintentional injuries other than transport injuries & 5.6 & 0.0 \\
\hline India & HIV/AIDS and tuberculosis & 5.3 & 8.3 \\
\hline India & Nutritional deficiencies & 5.1 & 2.1 \\
\hline India & Mental and behavioral disorders & 5.0 & 5.7 \\
\hline India & Other non-communicable diseases & 4.7 & 5.7 \\
\hline India & Ischemic heart disease & 4.6 & 15.6 \\
\hline India & Chronic obstructive pulmonary dise ase & 4.2 & 2.1 \\
\hline India & Musculoskeletal disorders & 3.9 & 2.1 \\
\hline India & Diabetes, urogenital, blood, and endocrine diseases & 3.7 & 12.0 \\
\hline India & Tuberculosis & 3.6 & 1.0 \\
\hline India & Sepsis and other infectious disorders of the newborn baby & 3.5 & 0.0 \\
\hline India & $\begin{array}{ll}\text { Iron-deficiency anemia } \\
\end{array}$ & 3.1 & 1.6 \\
\hline India & Neoplasms & 3.0 & 32.8 \\
\hline India & Low back and neck pain & 2.9 & 0.0 \\
\hline India & Neurological disorders & 2.9 & 4.7 \\
\hline
\end{tabular}

\section{Table 2: India 2005, \% DALY and \% Clinical trials}

Table 2 shows that there is not a perfect match between \% clinical trials done in this period and DALYs. India needs more trials on communicable, neonatal and nutritional diseases, also on diseases like diarrhea, respiratory infections and other common infections.

Graph 2 shows the correlation between \% DALYs and \% Clinical trials. We calculated Pearson Correlation Coefficient and the value of $\mathrm{R}$ is 0.6811 . This shows a moderate positive correlation, which means there is a tendency for high \% DALY variable scores go with high \% Clinical Trial variable scores (and vice versa). P Value from Pearson (R) is 0.000178 . The result is significant at $p<0.05$. 


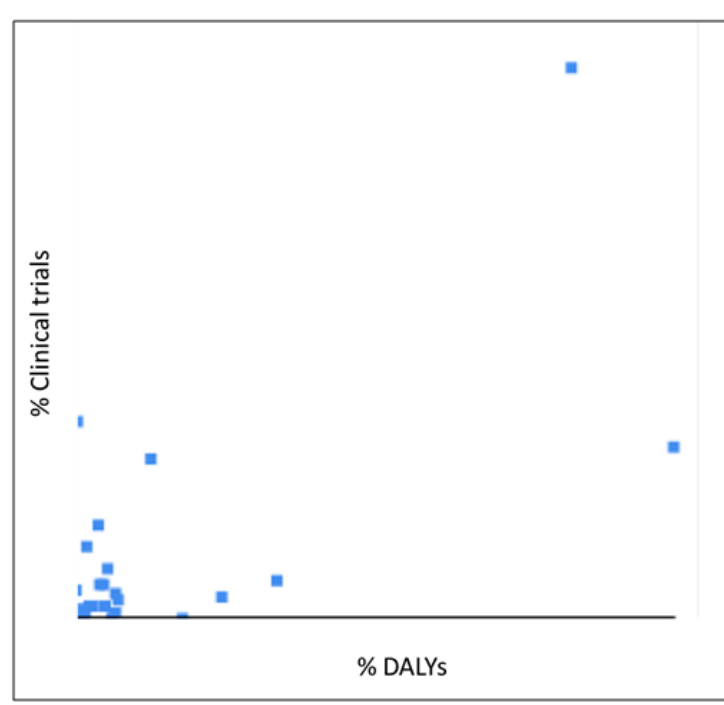

\section{Graph 2: Correlation between \% DALYs and \% Clinical Trials for India 2005}

Table 3 shows the values of \% of DALYS and \% Clinical trials done in Germany in 2010 for top 25 diseases in Germany. ${ }^{4,5}$ This table clearly shows that non communicable diseases $(88.1 \%)$, Cardiovascular and circulatory diseases (19.4\%) and Neoplasms (17.8\%) are the top 3 diseases in this period. There is not much change in 2010 DALYs as compared to 2005 data.

\begin{tabular}{|c|c|c|c|}
\hline Location & Cause & $\%$ DALYS & $\%$ clinical trials \\
\hline Germany & Total (All Causes) & 100 & 100 \\
\hline Germany & Non-communicable diseases & 88.1 & 95.3 \\
\hline Germany & Cardiovascular and circulatory diseases & 19.4 & 28.2 \\
\hline Germany & Neoplasms & 17.8 & 23.1 \\
\hline Germany & Musculoskeletal disorders & 15.8 & 4.6 \\
\hline Germany & Low back and neck pain & 11.5 & 0.7 \\
\hline Germany & Mental and behavioral disorders & 11.4 & 5.1 \\
\hline Germany & Ischemic heart disease & 10.5 & 14.3 \\
\hline Germany & Low back pain & 9.0 & 0.4 \\
\hline Germany & Injuries & 8.4 & 0.1 \\
\hline Germany & Diabetes, urogenital, blood, and endocrine diseases & 6.2 & 10.4 \\
\hline Germany & Neurological disorders & 4.8 & 5.4 \\
\hline Germany & Other non-communicable diseases & 4.8 & 6.2 \\
\hline Germany & Unipolar depressive disorders & 4.6 & 1.0 \\
\hline Germany & Unintentional injuries other than transport injuries & 4.5 & 0.1 \\
\hline Germany & Chronic respiratory diseases & 4.5 & 5.9 \\
\hline Germany & Major depressive disorder & 3.9 & 1.0 \\
\hline Germany & Cerebrovascular disease & 3.9 & 2.3 \\
\hline Germany & Trachea, bronchus, and lung cancers & 3.6 & 3.0 \\
\hline Germany & Communicable, maternal, neonatal, and nutritional disorders & 3.5 & 11.6 \\
\hline Germany & Falls & 3.4 & 0.1 \\
\hline Germany & Chronic obstructive pulmonary disease & 2.9 & 2.7 \\
\hline Germany & Diabetes mellitus & 2.7 & 7.9 \\
\hline Germany & Other musculoskeletal disorders & 2.5 & 0.0 \\
\hline Germany & Neck pain & 2.5 & 0.3 \\
\hline Germany & Ischemic stroke & 2.4 & 2.2 \\
\hline
\end{tabular}

Table 3: Germany 2010, \% DALY and \% Clinical trials 
It is evident that the \% clinical trials done in this period there is a good match between \% DALYs and \% Clinical trials.

Graph 3 shows the correlation between \% DALYs and \% Clinical trials. We calculated Pearson Correlation Coefficient and the value of $R$ is 0.96 . This shows a strong positive correlation, which means that high \% DALY variable go with high \% Clinical trial variable scores (and vice versa). $\mathrm{P}$ Value from Pearson $(R)$ is $<0.00001$. The result is significant at $\mathrm{p}<0.05$.

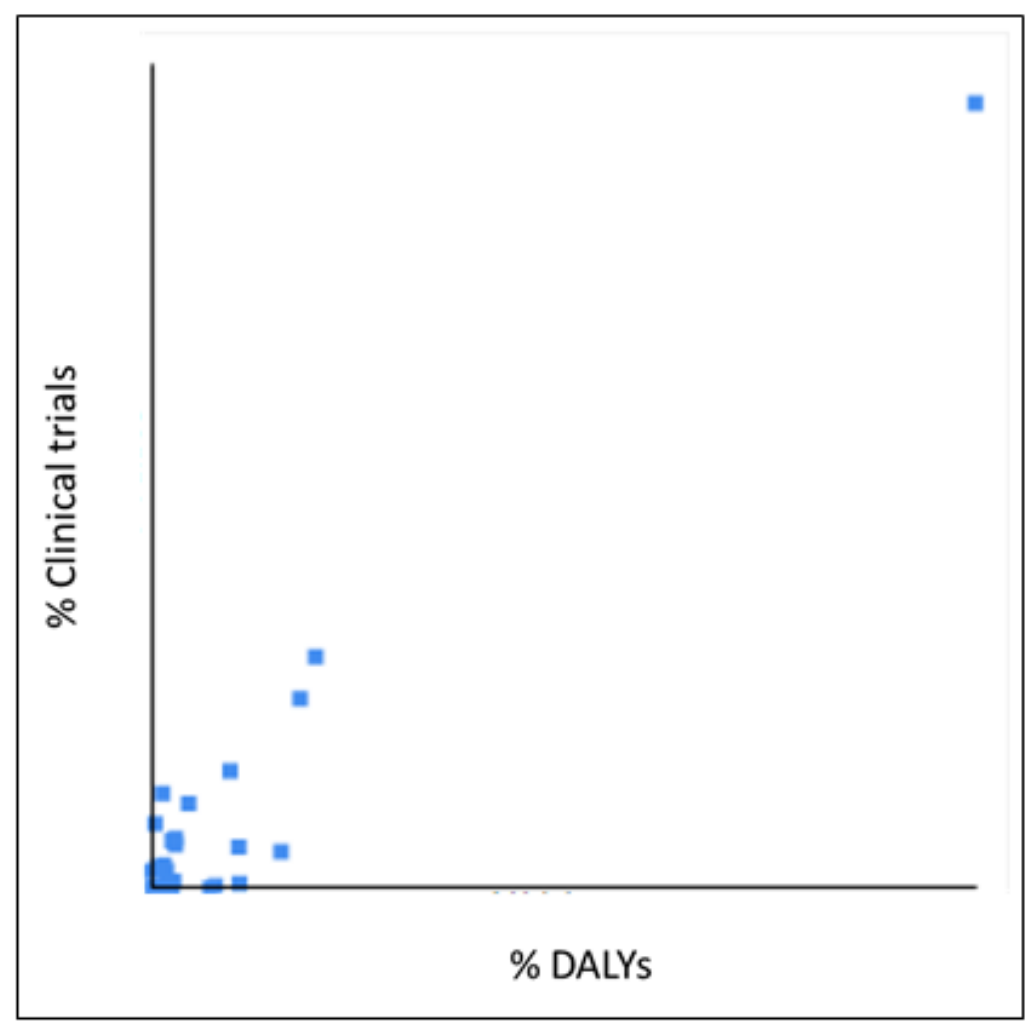

\section{Graph 3: Correlation between \% DALYs and} $\%$ Clinical Trials for Germany 2010

Table 4 shows the values of \% of DALYS and \% Clinical trials done in India in 2010 for top 25 diseases in India. ${ }^{4} 5$ This table clearly shows that non communicable diseases (45.4\%), Communicable, maternal, neonatal and nutritional disorders (42.7\%) and Diarrhea, lower respiratory infections and other common infections (15.4\%) are the top 3 diseases in this period. It shows clearly that non-communicable diseases are at top position which was not the case in 2005. 
REVIEW ARTICLE

\begin{tabular}{|c|c|c|c|}
\hline Location & Cause & $\%$ DALYS & \% clinical trials \\
\hline India & Total (All Causes) & 100.0 & 100.0 \\
\hline India & Non-communicable diseases & 45.4 & 91.5 \\
\hline India & $\begin{array}{r}\text { Communicable, maternal, neonatal, and nutritional } \\
\text { disorders }\end{array}$ & 42.7 & 22.4 \\
\hline India & $\begin{array}{r}\text { Diarrhea, lower res pira tory infections, meningitis, and other } \\
\text { common infectious diseases }\end{array}$ & 15.4 & 8.5 \\
\hline India & Neonatal disorders & 12.7 & 2.8 \\
\hline India & Injuries & 11.8 & 0.0 \\
\hline India & Cardiovascular and circulatory diseases & 9.4 & 25.5 \\
\hline India & Chronic respiratory diseases & 6.9 & 3.5 \\
\hline India & Unintentional injuries other than trans port injuries & 5.7 & 0.0 \\
\hline India & Mental and behavioral disorders & 5.6 & 6.2 \\
\hline India & Preterm birth complications & 5.5 & 0.4 \\
\hline India & Diarrheal diseases & 5.2 & 2.5 \\
\hline India & Lower respiratory infections & 5.2 & 2.4 \\
\hline India & Ischemicheart disease & 5.0 & 11.0 \\
\hline India & Chronic obstructive pulmonary disease & 5.0 & 1.4 \\
\hline India & Other non-communicable diseases & 4.9 & 5.7 \\
\hline India & HIV/AIDS and tuberculosis & 4.8 & 5.5 \\
\hline India & Nutritional deficiencies & 4.6 & 1.1 \\
\hline India & Musculoskeletal disorders & 4.5 & 4.1 \\
\hline India & Diabetes, urogenital, blood, and endocrine diseases & 4.1 & 18.2 \\
\hline India & Neoplasms & 3.7 & 20.4 \\
\hline India & Neurological disorders & 3.4 & 4.6 \\
\hline India & Low back and neck pain & 3.3 & 0.6 \\
\hline India & Sepsis and other infectious disorders of the newborn baby & 3.2 & 0.6 \\
\hline India & Transport injuries & 3.1 & 0.0 \\
\hline India & Self-harm and interpersonal violence & 3.0 & 0.0 \\
\hline
\end{tabular}

Graph 4 shows the correlation between \% DALYs and \% Clinical trials. We calculated Pearson Correlation Coefficient and the value of $\mathrm{R}$ is 0.7637 . This shows a strong positive correlation, which means that high DALYs variable scores go with high \% Clinical trials scores (and vice versa). $P$ Value from Pearson $(R)$ is $<0.00001$. The result is significant at $p<0.05$. 


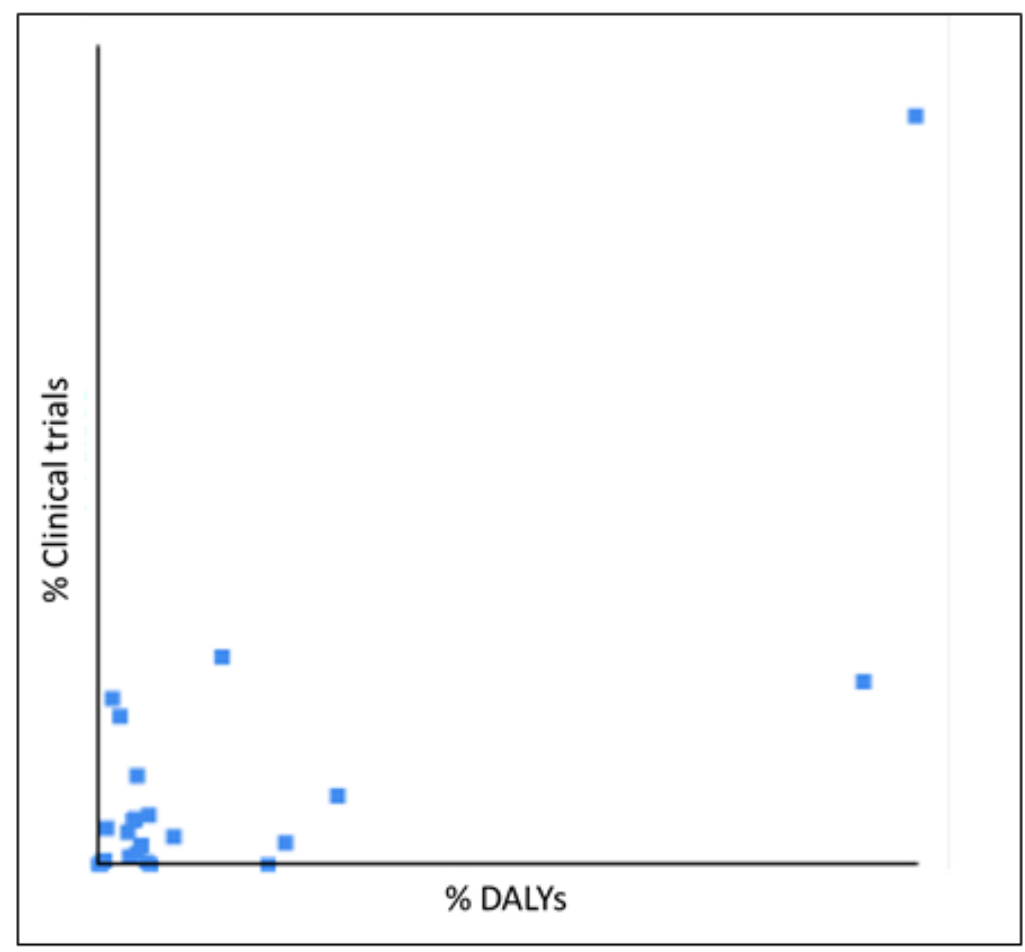

Graph 4: Correlation between \% DALYs and $\%$ Clinical Trials for India 2010

CONCLUSION: Our findings indicate that there was a good correlation between the \% DALYS and \% Clinical trials for Germany in 2005 and 2010. Emphasis of clinical research during this period was put on the top 25 diseases in Germany. For India in 2005 there was a moderate positive correlation, which means there is a tendency for high \% DALY variable scores go with high \% Clinical Trial variable scores (and vice versa). During this period there was a need in India to have new drug through more trials on communicable, neonatal and nutritional diseases, also on diseases like diarrhea, respiratory infections and other common infections. 2010 data showed the improvement in India in terms of match between \% DALYs and \% Clinical Trials. But careful observation of the data shows still a need for more trials on Communicable, maternal, neonatal and nutritional disorders.

Implications for policymakers and the future: India like most of the developing countries cannot fully afford to support medical research. In addition, the pharmaceutical industry may be reluctant to sponsor trials in the developing world because the prospects for profit are limited, even if effective treatments are developed. Not for profit organizations and medical institutes may also have difficulty supporting such research on their own. They may have difficulty in setting priorities and often prefer to offer practical help rather than foster research. Despite these limitations, the major health problems of the developing world cannot be set aside. Their consequences also affect the developed world. Success can often be obtained with limited funds. Many diseases in India coexist in vulnerable patient groups, and treatment or prevention of one 
disease may have a major effect on another (for example, nutritional disorders, infections and maternal-neonatal disorders). Seemingly expensive interventions can become affordable if there is strong political will and collaboration with the industry. The pace of clinical research is already at the top as India has become major destination for global clinical research. Still there is a need for more international support and focus on country specific diseases "local diseases and disorders" instead of fostering just another form of colonization. It is certain that Indian researchers should have a meaningful say in setting research priorities, and outside support should help develop sustainable local research capacity.

\section{REFERENCES:}

1. Rochon P. CMAJ. 2004; 170 (11): 1673-1677

2. Economic Times. 2013. Need for more conducive environment for clinical trials: ISCR

3. Petryna A. Ethical variability: Drug development and globalizing clinical trials. American Ethnologist. 2005; 32(2): 183-197.

4. Institute for Health Metrics and Evaluation. 2014. (http: //www.healthdata.org/search-gbddata)

5. A service of the U.S. National Institutes of Health. ClinicalTrials.gov

\section{AUTHORS:}

1. Dnyanesh Limaye

2. Gerhard Fortwengel

3. Vaidehi Limaye

\section{PARTICULARS OF CONTRIBUTORS:}

1. Professor, Department of Pharmacology, University of Applied Sciences and Arts, Hannover, Germany.

2. Professor, Department of Pharmacology, University of Applied Sciences and Arts, Hannover, Germany.

3. Professor, Department of Pharmacology, University of Mumbai, India.

\section{NAME ADDRESS EMAIL ID OF THE CORRESPONDING AUTHOR: \\ Dr. Vaidehi Limaye, 12/595, Srinivas (Kesar-Villa), Dr. Ambedkar Road, Matunga East, Mumbai - 400019. \\ E-mail: vaidehi1in@yahoo.com}

Date of Submission: 24/11/2014. Date of Peer Review: 25/11/2014. Date of Acceptance: 15/12/2014. Date of Publishing: 29/12/2014. 